\title{
A novel scaling indicator of early warning signals helps anticipate tropical cyclones
}

Article

Published Version

Creative Commons: Attribution 4.0 (CC-BY)

Open Access

Prettyman, J., Kuna, T. and Livina, V. (2018) A novel scaling indicator of early warning signals helps anticipate tropical cyclones. Europhysics Letters, 121 (1). 10002. ISSN 02955075 doi: https://doi.org/10.1209/0295-5075/121/10002 Available at https://centaur.reading.ac.uk/75939/

It is advisable to refer to the publisher's version if you intend to cite from the work. See Guidance on citing.

To link to this article DOI: http://dx.doi.org/10.1209/0295-5075/121/10002

Publisher: Institute of Physics Publishing

All outputs in CentAUR are protected by Intellectual Property Rights law, including copyright law. Copyright and IPR is retained by the creators or other copyright holders. Terms and conditions for use of this material are defined in the End User Agreement.

\section{www.reading.ac.uk/centaur}

\section{CentAUR}

Central Archive at the University of Reading

Reading's research outputs online 
Related content Effects of quantization on detrended
fluctuation analysis Zhu Song-Sheng, Xu Ze-Xi, Yin Kui-Xi et al.

Correlation between detrended fluctuation analysis and the Lempel-Ziv complexity in nonlinear time series analysis

Tang You-Fu, Liu Shu-Lin, Jiang Rui-Hong et al.

- A study of the early warning signals of abrupt change in the Pacific decadal oscillation

Wu Hao, Hou Wei, Yan Peng-Cheng et al. 


\title{
A novel scaling indicator of early warning signals helps anticipate tropical cyclones
}

\author{
J. Prettyman ${ }^{1,2}$, T. KunA ${ }^{1}$ and V. Livina ${ }^{2}$ \\ 1 University of Reading - Whiteknights, Reading, RG6 6AX, UK \\ 2 National Physical Laboratory - Hampton road, Teddington, TW11 0LW, UK
}

received 8 November 2017; accepted in final form 9 February 2018

published online 5 March 2018

PACS 05.45.Tp - Time series analysis

PACS 05.45.-a - Nonlinear dynamics and chaos

PACS 02.30.0z - Bifurcation theory

\begin{abstract}
Tipping events in dynamical systems have been studied in many contexts, often modelled by the decay of critical modes, system states which are tending towards bifurcation, characterised by increased return times to stable equilibria. Temporal scaling properties of time series data can be used to detect the presence of a critical mode by estimating the decay rate, and indicators of changes in these properties may therefore be used to provide an early warning signal (EWS) for an impending tipping event. The lag-1 autocorrelation function $(\mathrm{ACF}(1))$ indicator and the detrended fluctuation analysis (DFA) indicator have previously been used in such a way; in this paper we introduce a novel scaling indicator based on the decay rate of the power spectrum (PS). We compare the ACF(1), DFA- and PS-indicators using artificial data; data from a model which includes a bifurcation point; and sea-level pressure data along the paths of 14 tropical cyclones. By using the PS-indicator with such data, we show that the new indicator may be used to provide an EWS in a context where the ACF(1)- and DFA-indicators fail.

Copyright (C) EPLA, 2018
\end{abstract}

Introduction. - Tipping events have been studied in many climatological and ecological contexts [1-6]. These tipping points, which may be thought of as qualitative changes in the underlying dynamical systems, have been modelled by the decay of stable equilibria [7], which we refer to as critical modes as they approach the critical threshold leading to a bifurcation. They are identified by their rate of decay, known as critical slowing-down, which can be estimated from the scaling properties of the system time series in the proximity of the tipping point.

The authors of [7] associate critical slowing-down with increasing return times to the critical mode, which is modelled as an autoregressive process and thus the lag- 1 autocorrelation function (ACF) is used to detect the critical slowing-down. The lag-1 ACF, or $\mathrm{ACF}(1)$, can therefore be used as a predictive indicator of tipping points [8], or early warning signal.

Furthermore, due to the scaling properties, it is possible to use longer-term autocorrelations of a time series as an indicator, the power-law decay rate of the ACF with increasing time lag is one such example. Detrended fluctuation analysis (DFA) averages the variance over non-overlapping segments of length $s$ of a time series after subtracting an order $n$ polynomial trend [9].
Since during the critical slowing down of the system dynamics DFA provides a method to study the scaling properties, similarly to the ACF, the DFA scaling indicator has been introduced as an alternative to the $\mathrm{ACF}(1)$ indicator [10]. Both the ACF(1)- and DFA-indicators have been shown to detect early warning signals of bifurcations and tipping points in a variety of systems [11,12].

In this paper we propose a novel scaling indicator based on the power-law decay rate of the power spectrum (PS), as the PS scaling exponent is related to the ACF and DFA scaling exponents [9]. We introduce the PS-indicator, then compare the performance of the PSindicator to the $\mathrm{ACF}(1)$ - and DFA-indicators using an artificially constructed time series. This allows us to compare the responses to changing scaling exponent $\beta$. We further compare the $\mathrm{ACF}(1)-$, DFA- and PS-indicators when applied to a time series from a classic example of a model with a pitchfork bifurcation, where we would at least expect that the $\mathrm{ACF}(1)$-indicator would provide a very clear EWS. We then apply the three scaling indicators to sea-level pressure data along the paths of 14 tropical cyclones, in order to assess the suitability of these methods, particularly the PS-indicator, in providing an EWS in such a geophysical context. In performing these experiments 
using artificial and real geophysical data, we aim to show that the new PS-indicator performs similarly to the other well-studied indicators, as we expect based on the analytical relationship [9]. Finally, we comment on the sensitivity of the PS-indicator method to the window-size parameter.

Scaling properties of time series. - Scaling properties of time series can be measured using three techniques: the autocorrelation function (ACF), detrended fluctuation analysis (DFA) and the power spectrum $[9,13]$. These three methods are illustrated in fig. 1 where we plot a time series of artificial data and three scaling curves. The data is a length 10000 red-noise series, with power spectrum scaling exponent of $\beta=0.85$. To generate this series, we first sample from a Gaussian distribution to approximate white noise, $X(t)$, we then take the fast Fourier transform of this white noise series (denoted $\hat{X}(f)$ ) and then transform this by

$$
\hat{Y}(f):=\hat{X}(f) \sqrt{f-\beta} .
$$

Finally, we use the inverse fast Fourier transform to obtain the time series $Y(t)$, which has power spectrum $S(f)$ proportional to $f^{-\beta}$.

The ACF scaling exponent $(\gamma)$, fig. 1(b), is the powerlaw decay rate of the autocorrelation function with increasing lag $(s)[9]$. Denote by $C(s)$ the autocorrelation with lag $s$ of time series $X$, then the scaling is defined as

$$
C(s) \sim s^{-\gamma},
$$

for long-range correlations. Following [10], we measure the exponent in the range $10 \leq s \leq 100$. For short-range correlated data, $C(s)$ decays exponentially and only $\mathrm{ACF}(1)$ is indicative of data variability in proximity to a tipping point.

The DFA scaling exponent $(\alpha)$ is measured following [9], fig. $1(\mathrm{c})$. Where $F(s)$ is the DFA function of time series $X$, with segment length (scale) $s$, we have

$$
F(s) \sim s^{\alpha}
$$

In this paper we consistently use an order-2 polynomial during the detrending step of DFA. Following the approach of [10], we measure the DFA exponent in the temporal range $10 \leq s \leq 100$, which is sensitive to changes in the system dynamics similarly to $\mathrm{ACF}(1)$.

The power spectrum scaling exponent $(\beta)$ is calculated by estimating the slope of the power spectrum $S(f)$ of the data, plotted on logarithmic axes [13], from which one can obtain $\beta$ via the scaling relationship

$$
S(f) \sim f^{-\beta} .
$$

Here, the power spectrum is approximated by the periodogram, obtained from the absolute value of the fast Fourier transform. We measure the exponent (slope) inside the frequency range $10^{-2} \leq f \leq 10^{-1}$ corresponding to the time range of 10 to 100 units, to put the technique in agreement with with the ACF and DFA methods, as above.
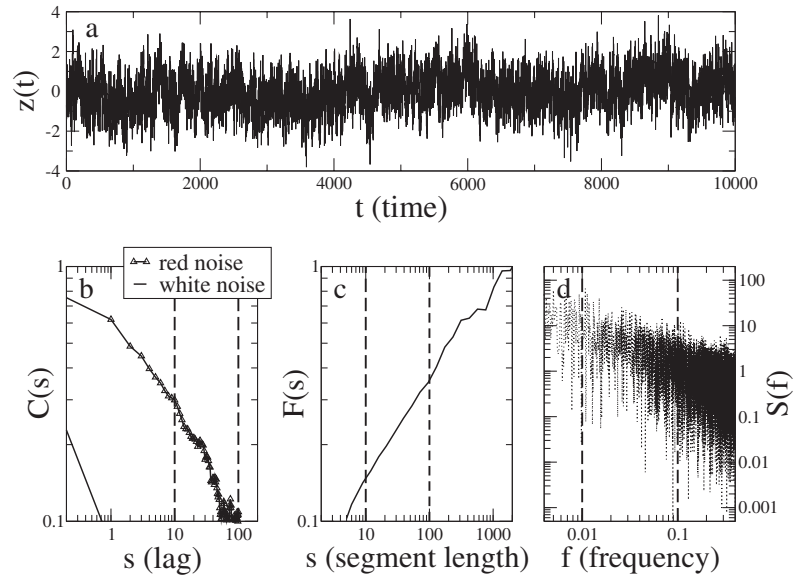

Fig. 1: Analysis of artificial red noise with scaling exponents measured using three different methods. (a) Red noise with a power spectrum scaling exponent of 0.85 . (b) The ACF of the red noise data is calculated for different lags and the exponent (negative slope) measured in the range $10 \leq s \leq 100$ (dashed lines). We note that the $\mathrm{ACF}(1)$-indicator $(C(1))$ is 0.64 . The $\mathrm{ACF}$ of a white noise series is also plotted for comparrison, in this case $C(s)=0$ for $s \geq 1$ and the exponent is also zero. (c) DFA calculated for the data and the exponent (slope) measured in the range $10 \leq s \leq 100$. (d) The power spectrum of the data, and the exponent (negative slope) measured in the frequency range $10^{-2} \leq f \leq 10^{-1}$.

Analytically, the three scaling exponents have the linear relationship:

$$
\alpha=\frac{1+\beta}{2}=1-\frac{\gamma}{2}
$$

in the asymptotic range [9]. We therefore expect that a measurement of $\beta$ could be used in an application where $\alpha$ or $\gamma$ are conventionally used, to provide an alternative metric for EWS, which may be informative in cases where other indicators fail to detect an approaching tipping point.

PS-indicator applied to artificial data. - In order to estimate an early warning signal for a tipping point, we calculate the value of an indicator in a sliding window of length $N$ points along the time axis, in the range $t_{1} \leq$ $t \leq t_{N}$, then in the range $t_{2} \leq t \leq t_{N+1}$, and so on, thus following the example of [8] where the ACF(1)-indicator (a measurement of lag-1 ACF, distinct from the ACF scaling exponent $\gamma$ ) is used. In that paper, the length of the window is chosen to be $10 \%$ of the length of the time series. In order to quantify uncertainties we will consider a range of window lengths. We first, however, take a fixed window length of 100 points in order to compare our PS-indicator to the ACF(1)-indicator used by [8] and the DFA-indicator used by [10], when applied to artificial data, illustrated in fig. 2. This experiment is in agreement with an equivalent result in [14].

We construct artificial data of length $10^{4}$ points by stitching together 50 time series of 200 points. To generate each sub-series we use the same method as used for fig. 1 

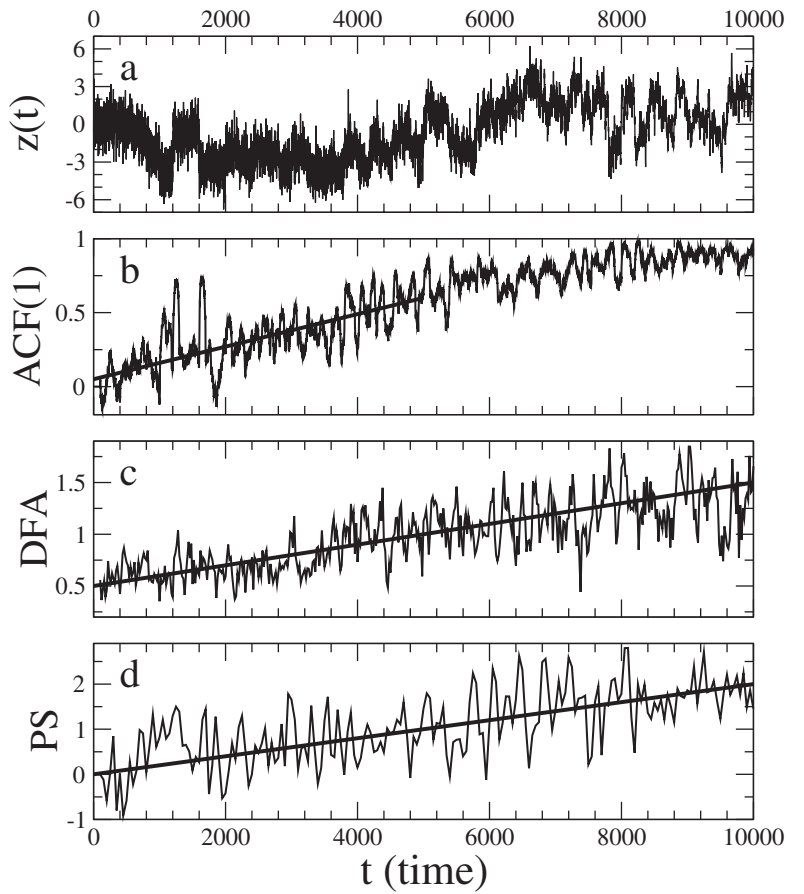

Fig. 2: Artificial data with ACF(1)-, DFA- and PS-indicators. (a) A time series is constructed by concatenating 50 sub-series of length 200 where the scaling exponent $\beta$ within each subseries is constant and increases over the whole series from 0 (white noise) to 2. (b)-(d) The ACF(1)-, DFA- and PSindicator methods are applied with window size 100. As the PS-indicator increases from 0 to 2, the $\mathrm{ACF}(1)$-indicator increases from 0 to 1 , and the DFA scaling exponent $\alpha$ increases from 0.5 to 1.5. Lines are added to show the linear trends; note that the $\mathrm{ACF}(1)$-indicator does not increase linearly as it approaches 1 .

(see eq. (1)) with values of $\beta$ such that the value varies linearly from 0 (no transformation, equivalent to white noise) to 2 [15]. We expect, therefore, that the PS-indicator $\beta$ will increase linearly from 0 to 2 . Because of the relationship between the indicators we also expect that the DFAindicator will increase linearly, as demonstrated in fig. 2. We have chosen this artificial time series, rather than, for example, the Ornstein-Uhlenbeck process [16-18], to provide an example specifically suited to the PS-indicator and to reflect the result in [14] where this series was used.

PS-indicator applied to model data. - We now apply the PS-indicator to a system including a bifurcation point, expecting that we will observe behaviour comparable to the $\mathrm{ACF}(1)$ - and DFA-indicators. We use a typical bifurcating system described by the stochastic differential equation

$$
\dot{z}(t)=-\frac{\partial}{\partial z}\left(z^{4}+\left(3-\frac{t}{200}\right) z^{2}\right)+\sigma \eta_{t},
$$

where $\sigma=0.05$ and the $\eta_{t}$ are independent samples from a Gaussian distribution. Equation (6) is integrated numerically with a time step of $\delta t=0.05$ from $t=1$ to $t=1000$, and sampled with $\Delta t=1$. In the range $t<600$ the system
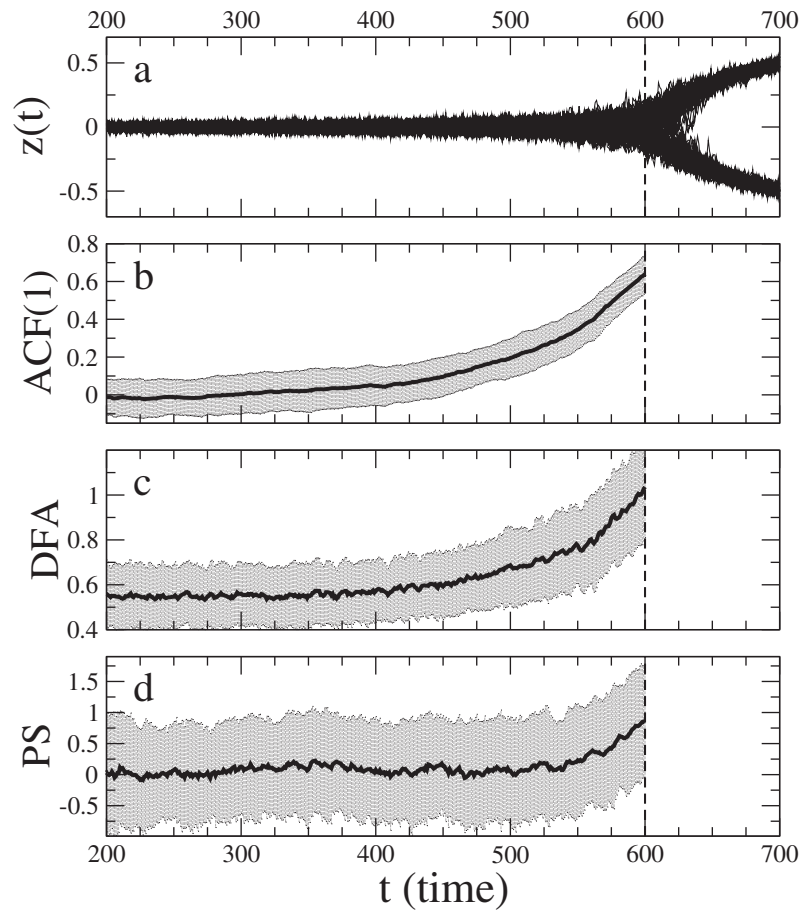

Fig. 3: ACF(1)-, DFA- and PS-indicators applied to model data. (a) Data from 100 runs of the model (see eq. (6)). (b)-(d) The mean $\operatorname{ACF}(1)$, DFA and PS-indicators (window size 100), with error bars of one standard deviation. The values of all the indicators begin to rise before the bifurcation, as expected, at around $t=500$, although it is most noticeable for the $\mathrm{ACF}(1)$-indicator.

has a single stable node $z=0$, the system then bifurcates after $t=600$ into a double-well potential with stable nodes at $z= \pm 0.05 \sqrt{t-600}, z=0$ becomes unstable.

The system is integrated 100 times to produce 100 time series of length 1000, see fig. 3(a). The EWS indicators are then applied to the 100 time series and the mean of all 100 indicator series is plotted with error bars of one standard deviation. We have performed this with the ACF(1)- and DFA-indicators (fig. 3(b), (c) and the new PS-indicator (fig. 3(d)). The ACF(1)-indicator provides a clear EWS as expected, there is a definite increasing trend starting at around $t=400$. We see a similar trend with the PSindicator, so the validity of the PS-indicator as an EWS is illustrated in principle. However, this indicator has high variance, and the mean at $t=600$ does not rise above the upper error bound (one standard deviation, $1 \sigma$ ) for $t<500$. This means that at the early development of the trend in the PS-indicator in live monitoring of this dynamical system, the rise of the indicator may be attributed to its stochastic variability.

It is necessary to note that the pitchfork bifurcation was chosen because in this case $\mathrm{ACF}(1)$ provides a clear EWS. Therefore, it is a good testbed for the PS-indicator, by analogy.

PS-indicator applied to tropical cyclones. - We now apply the PS-indicator to data from a real geophysical 

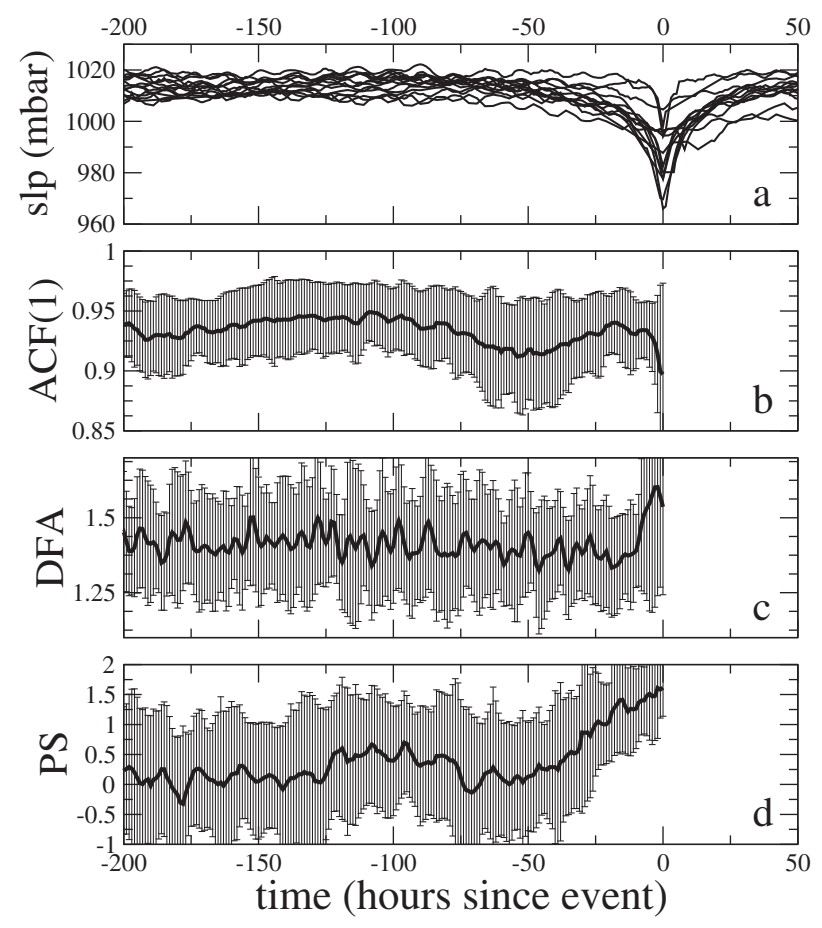

Fig. 4: $\mathrm{ACF}(1)-$, DFA- and PS-indicator methods applied to sea-level pressure data. (a) Data from the 14 tropical cyclones. (b)-(d) The mean ACF(1)-, DFA- and PS-indicators (window size 100), with error bars of 1 standard deviation. The ACF(1)indicator does not provide a clear EWS in this case. The DFAindicator shows a noticable increasing trend just before the event. The PS-indicator appears to rise around 50 hours before the cyclone event.

system. We use hourly, one-dimensional sea-level pressure data from observation stations within $2 \mathrm{~km}$ of the landfall locations of 14 tropical cyclones. The data is obtained from the HadISD2005 dataset, available from the UK MetOffice [19]. The 14 cyclones were selected for falling close to one of these stations, and being ranked category 4 or 5 , using the Saffir-Simpson scale, at the time of landfall. The 14 selected are: Atlantic hurricanes Andrew (1992), Opal (1995), Floyd (1999), Charley (2004), Frances (2004), Jeanne (2004), Katrina (2005), Rita (2005), Ivan (2006) and Ernesto (2006); typhoons Zeb (1998), Megi (2010) and Flo (1990); and Cyclone Hudhud (2014).

We do not attempt to provide an analytical description of the tropical cyclone data and it does not appear to contain a bifurcation in the same sense as the pitchfork bifurcation (eq. (6), fig. 3). However, there is a transitional tipping point in the sense that the pressure drops suddenly when the cyclone passes over, and this most likely affects the dynamics of the fluctuations, providing a detectable EWS. We center the time series for each cyclone on the minimum pressure value (fig. 4(a)), which time we call $t=0$. This cannot be called analogous to the bifurcation point at $t=600$ in the pitchfork bifurcation example, but is a common feature which is convenient to use for the purpose of comparison. We use the same method as used for the bifurcating model data in the previous section, applying the indicator in a sliding window of 100 points to all 14 time series and plotting the mean of all 14 indicator series, with error bars of one standard deviation. Again, we apply both the ACF(1)- and DFA-indicators (fig. 4(b),(c)) and the new PS-indicator (fig. 4(d)). The $\mathrm{ACF}(1)$-indicator, in this case, does not appear to provide a clear EWS, whereas the PS-indicator does show an increasing trend starting at around 50 hours before the minimum pressure.

Sensitivity of the method to window size. - The method described provides an estimate of the changes in the PS scaling exponent in a window of 100 points. It is desirable to use a very large number of points to give an accurate estimate of the scaling exponent from the periodogram, less sensitive to periodic oscillations in the data and also with less variability over time. However, one must compromise this since the window size must correspond to the time scale of the phenomenon under investigation; of course there is also a limit imposed by the length of the time series data. We require a window size such that the indicator variance is significantly small that we can see the EWS, but that the rise in the indicator value before the tipping event is large enough so that we can recognise it as an EWS.

It is useful to produce a contour plot of the mean value of the indicator over time, with window size on the $y$-axis. We expect that the EWS apparently becomes stronger with decreasing window size, above a certain size where the variability becomes so large that it is not possible to detect an EWS. In this way, we make a subjective choice as to the optimum window size to use in each context and assume that we can reasonably expect that this choice can be applied to new data from the same source.

In fig. 5 we see that there is an increasing trend in the PS-indicator towards the end of the time series with almost all window sizes. The exceptions occur periodically where the window size is approximately $12 n \pm 1$ for integer $n$, using these window sizes the indicator is approximately 1.5 over the whole series. Note that the most obvious signals occur with window sizes 102, 114 and 126 . Using larger values (e.g., 174) the useful variability in the indicator is smoothed out so that the trend towards the end of the series is less obvious.

The periodic pattern in the contour plot (fig. 5) is caused by an oscillation with period $\approx 12$ hours in the sea-level pressure data, likely caused by tidal influences. In this context it is therefore essential to consider the sensitivity of the method to the window size parameter, as we have done here.

Discussion. - We have developed a novel indicator of EWS for tipping points in dynamical systems by estimating the power-law decay rate of the power spectrum in a sliding window. We have applied this PS-indicator to 


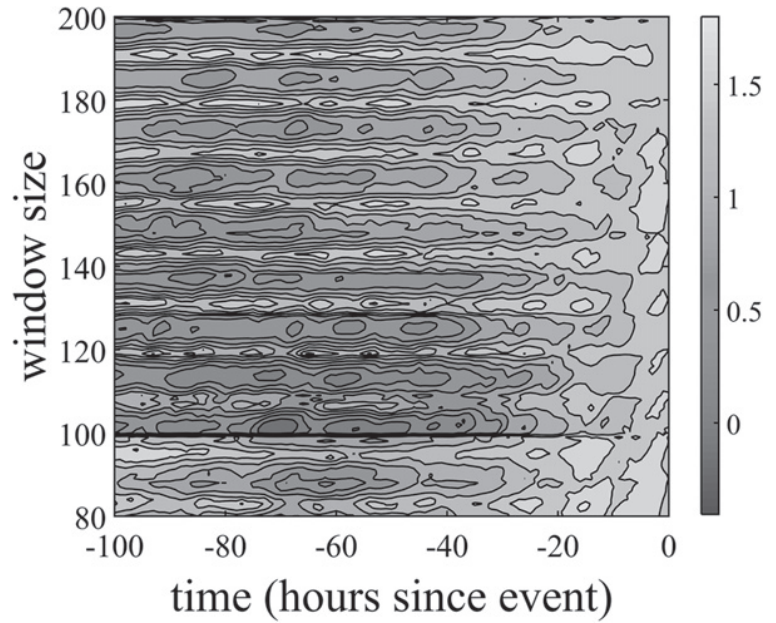

Fig. 5: Mean PS-indicator for tropical cyclone data (see fig. 4) calculated using window sizes from 80 to 200 . The PS-indicator appears to rise around 40 hours before the cyclone event in almost all cases, the exceptions occur when the window size has the value $12 n \pm 1$, in these cases the indicator is high (approximately 1.5) over the entire series.

three time series: an artificial example of increasing scaling exponent; a model including a bifurcation; and sea-level pressure data measured at the landfall sites of 14 tropical cyclones.

In the case of high-frequency sampling of data, it is likely that the autocorrelation would be fluctuating at very high level (close to maximal value 1 ) and not providing any meaningful EWS information. In such cases, for applying any EWS indicator based on scaling properties (ACF, DFA or PS), it would be useful to pre-process the data with some aggregation or averaging, similarly to [8]. However, this issue is not specific to the new PSindicator. Moreover, in many cases a time series is preprocessed before calculating EWS indicators (for instance, using Gaussian filter or moving average), which immediately changes the absolute value of autocorrelation and other scaling exponents. This means that the values of autocorrelation change, and it is no longer bounded by the critical value 1 . In such cases, the increasing trend of an EWS indicator is more informative than its values, which is a shortcoming of the approach with such data preprocessing. So far, its impact on the EWS indicators has not been studied, either in the context of analytical rigour or in the context of proximity to a tipping point; this must be a topic of further research in the area of early warning signals.

On the other hand, the length of the series (always finite and often imposing finite-size-effect challenges) may be problematic in case of estimation, for instance, variance in fixed-size windows. As was demonstrated in fig. 2 (middle panel) of [14], using fixed-size window in studying a dataset with increasing scaling exponent may generate a decreasing variance indicator.
We have found that the PS-indicator behaves similarly to the previously used $\mathrm{ACF}(1)$ - and DFA-indicators applied to the model data, but with larger variance, which limits its usefulness in situations where the model cannot be observed multiple times. When applied to the sealevel pressure data, the $\mathrm{ACF}(1)$ - and DFA-indicators do not appear to provide an EWS. However, the PS-indicator clearly does begin to increase around 50 hours before the lowest pressure, about the same point that the decreasing trend in pressure becomes visible, suggesting that it can provide a detectable EWS in this context.

The approaching cyclone is chosen as an example of a system which has not previously been studied using tipping-point EWS methods, and for which the dynamics of the system are unknown, thus providing a "blind" experiment. The passing cyclone is not modelled as a phase transition, and it is not known whether there exists a critical point such that we might claim universality of the scaling exponents. Rather, we have a time series which superficially resembles other geophysical tippingpoint examples (see [11]), thus we wish to experiment with our existing EWS methods, and then compare the performance of our new indicator. In this sense, it is a heuristic investigation of the properties of the new technique, with comparison with similar methods. We report our findings to facilitate further investigation of this technique.

We conclude that the PS-indicator is a novel and useful technique which behaves similarly to the related $\mathrm{ACF}(1)-$ and DFA-indicators in idealised systems. It also shows signs of providing a useful EWS for the real geophysical system of impending tropical cyclones, where the ACF(1)indicator fails.

\section{REFERENCES}

[1] Scheffer M., Carpenter S., Foley J. A., Folke C. and Walker B., Nature, 413 (2001) 591.

[2] Livina V., Kwasniok F., Lohmann G., Kantelhardt J. and Lenton T., Clim. Dyn., 37 (2011) 2437.

[3] Veraart A. J., FaAssen E. J., Dakos V., van Nes E. H., Lürling M. and Scheffer M., Nature, 481 (2012) 357.

[4] Ashwin P., Wieczorek S., Vitolo R. and Cox P., Philos. Trans. R. Soc. London, Ser. A: Math. Phys. Eng. Sci., 370 (2012) 1166.

[5] Gsell A. S., Scharfenberger U., ÖZkundakci D., Walters A., Hansson L.-A., Janssen A. B., Nõges P., Reid P. C., Schindler D. E., Van Donk E. et al., Proc. Natl. Acad. Sci. U.S.A., 113 (2016) E8089.

[6] Ditlevsen P., Tipping Points in the Climate System, in Nonlinear and Stochastic Climate Dynamics, edited by Franzke Christian and O'Kane Terence (Cambridge University Press) 2017, pp. 33-53.

[7] Scheffer M., Bascompte J., Brock W. A., Brovkin V., Carpenter S. R., Dakos V., Held H., Van Nes E. H., Rietkerk M. and Sugihara G., Nature, 461 (2009) 53. 
[8] Held H. and Kleinen T., Geophys. Res. Lett., 31 (2004) L23207.

[9] Kantelhardt J. W., Koscielny-Bunde E., Rego H. H., Havlin S. and Bunde A., Physica A: Stat. Mech. Appl., 295 (2001) 441.

[10] Livina V. N. and Lenton T. M., Geophys. Res. Lett., 34 (2007) L03712.

[11] Dakos V., Scheffer M., van Nes E. H., Brovkin V., Petoukhov V. and Held H., Proc. Natl. Acad. Sci. U.S.A., 105 (2008) 14308.

[12] Lenton T., Livina V., Dakos V., Van Nes E. and Scheffer M., Philos. Trans. R. Soc. London, Ser. A: Math. Phys. Eng. Sci., 370 (2012) 1185.

[13] Bak P., Tang C. and Wiesenfeld K., Phys. Rev. A, 38 (1988) 364.
[14] Livina V., Ditlevsen P. and Lenton T., Physica A: Stat. Mech. Appl., 391 (2012) 485.

[15] Makse H. A., Havlin S., Schwartz M. and Stanley H. E., Phys. Rev. E, 53 (1996) 5445.

[16] Hasselmann K., Tellus, 28 (1976) 473.

[17] Ditlevsen P. D. and Johnsen S. J., Geophys. Res. Lett., 37 (2010).

[18] Ritchie P. and Sieber J., Chaos, 26 (2016) 093116.

[19] HADISD2005, Hadisd2005 dataset, ww. metoffice.gov . uk/hadobs/hadisd/, accessed: 2016-04-01; DunN R. J. H., Willett K. M., Thorne P. W., Woolley E., Durre I., Dai A., Parker D. E. and Vose R. S., Clim. Past, 8 (2012) 1649; Smith Adam, Lott Neal and Vose Russ, Bull. Am. Meteorol. Soc., 92 (2011) 704 . 